\title{
Diagnosis of bronchobiliary fistula by bilirubin crystallization in the alveolar lavage fluid: case reports and literature review
}

\author{
Nan Zhang ${ }^{1}$, Yan Chen ${ }^{2}$, Lihong Tang ${ }^{3}$, Daoyin Zhou ${ }^{2}$, Tianwen Hou ${ }^{1}$ \\ ${ }^{1}$ Clinical Laboratory of Hebei Chinese Traditional Hospital, Shijiazhuang, China; ${ }^{2}$ Clinical Laboratory of Changhai Hospital, Shanghai, China; \\ ${ }^{3}$ Clinical Laboratory of Urumchi Friendship Hospital, Urumchi, China \\ Correspondence to: Yan Chen. Department of Laboratory Diagnosis of Changhai Hospital Building, 5 Yangpu District of Shanghai, Shanghai 200433, \\ China. Email: chenyan770628@163.com.
}

\begin{abstract}
Bronchobiliary fistula (BBF) refers to the abnormal traffic between the biliary tract and the bronchus. The condition is very rare and usually develops secondary to liver echinococcosis or amebiasis, liver abscess, trauma, biliary obstruction, or tumors. BBF has a high mortality rate and currently, there are no accurate and effective diagnostic methods. This study reports the diagnosis and treatment of two patients with BBF which were confirmed by detecting bilirubin crystallization in the sputum. The first patient was a 45-year-old woman admitted to the hospital with "recurrent cough and lung infection". She had a history of multiple biliary tract surgeries and bilirubin crystallization was detected in bronchoalveolar lavage fluid (BALF) upon examination. Computed tomography (CT) imaging and magnetic resonance cholangiopancreatography (MRCP), together with clinical features, confirmed a diagnosis of BBF. The second patient was a 53-year-old woman admitted to the hospital with coughing and bile-like sputum. She had a history of cholangiocarcinoma surgery and bilirubin crystallization was detected in the cytomorphological BALF examination. Endoscopic retrograde cholangiopancreatography (ERCP) combined with clinical features confirmed a diagnosis of BBF. Both patients recovered after treatment and were discharged from the hospital. The clinical diagnosis of BBF largely relies upon imaging combined with clinical standards, and BALF examinations are rarely performed. This current investigation retrospectively analyzed the diagnosis and treatment of two cases of BBF, and demonstrated that bilirubin crystallization in the BALF may be an important diagnostic indicator for BBF.
\end{abstract}

Keywords: Bronchobiliary fistula (BBF); alveolar lavage fluid; bilirubin crystallization; cytomorphology; case report

Submitted Mar 24, 2021. Accepted for publication Jun 15, 2021.

doi: 10.21037/apm-21-1040

View this article at: https://dx.doi.org/10.21037/apm-21-1040

\section{Introduction}

Bronchobiliary fistula (BBF) refers to the abnormal traffic between the biliary system and the bronchial tree, through which bile can be coughed out of the body. It represents a serious complication of hepatobiliary diseases (1). BBF can be categorized into primary and secondary conditions (2). Primary BBF is very rare in clinical practice, while secondary BBF occurs secondary to trauma, surgery, biliary obstruction, tumors, or liver abscesses (3). The incidence of BBF after hepatobiliary surgery is $<0.1 \%$. However, the clinical manifestations of BBF are complicated, and most of the patients developed respiratory symptoms at first. At present, there is no efficient clinical method for the specific diagnosis of BBF. In fact, BBF is easily confused with other diseases such as lung infections, and this may lead to delays in diagnosis and treatment, thus increasing the mortality rate of such patients.

This paper retrospectively analyzed the diagnosis and treatment of two patients with BBF who were admitted to our hospital. Both patients were hospitalized with symptoms of respiratory tract infection and had a history of multiple 
biliary tract operations. As BBF is a complication caused by repeated biliary tract surgery, this was consistent with the common features of BBF. However, in contrast to previous reports, routine examination of the bronchoalveolar lavage fluid (BALF) showed bilirubin crystallization in both patients. The diagnosis of BBF was confirm in both patients by endoscopy and imaging examination. Therefore, the detection of bilirubin crystallization in the alveolar lavage fluid may have important diagnostic value in patients with BBF. We present the following article in accordance with the CARE reporting checklist (available at https://dx.doi. org/10.21037/apm-21-1040).

\section{Case presentation}

All procedures performed in studies involving human participants were in accordance with the ethical standards of the institutional and/or national research committee(s) and with the Helsinki Declaration (as revised in 2013). Written informed consent was obtained from the patient.

\section{Clinical data}

\section{Case 1}

A 45-year-old female was admitted to the outpatient clinic with "recurrent cough and lung infection". The patient had a previous intrahepatic bile duct lithotripsy and cholecystectomy at a local hospital in 2003 due to hepatolithiasis. After the operation, the stone was repeatedly extracted through the biliary drainage tube, and the biliary drainage tube was removed at the end of 2003. Thereafter, the patient underwent recurrent calculus of the bile duct with cholangitis, and she was repeatedly managed in the local hospital for infections with symptomatic and supportive treatments. In 2010, the patient underwent intrahepatic bile duct lithotripsy again in the local hospital and the stone was repeatedly extracted through the biliary drainage tube. Subsequently, the biliary drainage tube was removed due to acute renal failure. At the beginning of 2015, the patient received anti-infection and expectorant treatment at the local hospital due to recurrent cough and sputum. There was minimal improvement of symptoms, and she was admitted to Changhai Hospital for further treatment in October 2017. After admission, the patient's physical examination revealed a body temperature of $37.6{ }^{\circ} \mathrm{C}$, and a slight phlegm sound in the lower right lung. Anti-infection treatment was administered and the relevant checks were improved. At the same time, routine examination and cytomorphology inspection of the BALF were performed. The routine examination showed that the BALF was yellow and turbid, the total number of cells was $1,600 \times 10^{6}$ cells $/ \mathrm{L}$, and the number of nucleated cells was $810 \times 10^{6}$ cells/ $\mathrm{L}$. The nucleated cells were classified as $80 \%$ neutrophils, $2 \%$ lymphocytes, and $18 \%$ macrophages. The BALF cytomorphology revealed bilirubin crystallization and a large number of bacteria (Figure $1 A, B)$. Due to the BALF bilirubin crystallization, the source of the bile was investigated.

The patient's chest computed tomography (CT) scan showed the following: (I) lower right lung infection with mild bronchiectasis in both lower lungs, and possible local $\mathrm{BBF}$ in the lower right lung lesion; and (II) dilation of the intrahepatic bile duct with multiple stones, perihepatic effusion, and multiple low-density lesions in the liver. Bronchoscopy results demonstrated that the tracheal carina was sharp and smooth, the main bronchus and the left and right lobes of the bronchial mucosa were smooth, and the lumen was unobstructed. No new organisms were observed, and the yellow bile reflux was observed in the outer basal segment of the lower right lobe. Magnetic resonance cholangiopancreatography (MRCP) examination revealed biliary cirrhosis and splenomegaly. Furthermore, the changes in the top of the liver and the lower right lung were consistent with BBF and lower right lung pneumonia. Based on the examination results and clinical manifestations, the patient was eventually diagnosed with BBF.

The patient's symptoms improved after administration of human freeze-dried fibrinogen combined with silicone plugs, and endobronchial one-way valve (EBV) closure treatment. The patient was also treated for the infection. She was discharged 10 days later with normal body temperature and no sputum was observed. Since discharge, the patient has been followed up 3 times, and good recovery without recurrence was noted.

\section{Case 2}

A 53-year-old female presented to Changhai Hospital in August 2017 with coughing, bile-like sputum, and fever. She had a history of elevated carbohydrate antigen (Carbohydrate antigen 199, CA199) which was detected in January 2016. Cholangiocarcinoma was found on MRI examination of the bile duct. A complicated liver cancer resection (segments 1, 2, 3, 4) was performed in January 2016, and the postoperative pathology showed micro-left 

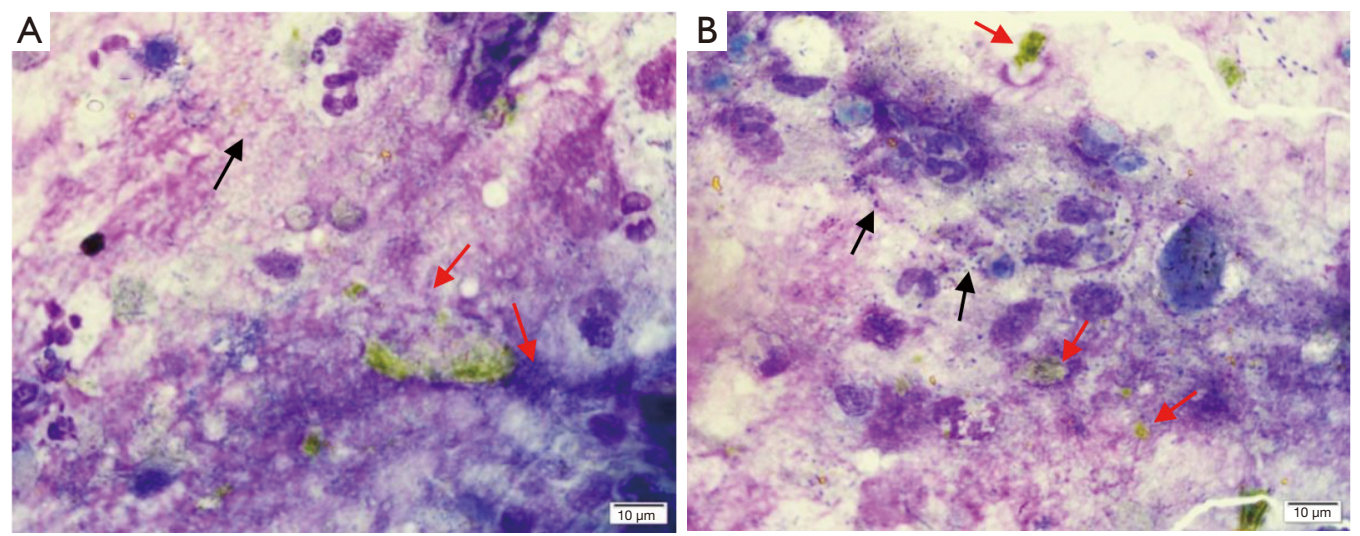

Figure 1 St. Regis staining of BALF smears $(\times 1,000$ magnification). Bilirubin crystallization is shown by the red arrows and bacteria is shown by the black arrows. BALF, bronchoalveolar lavage fluid.
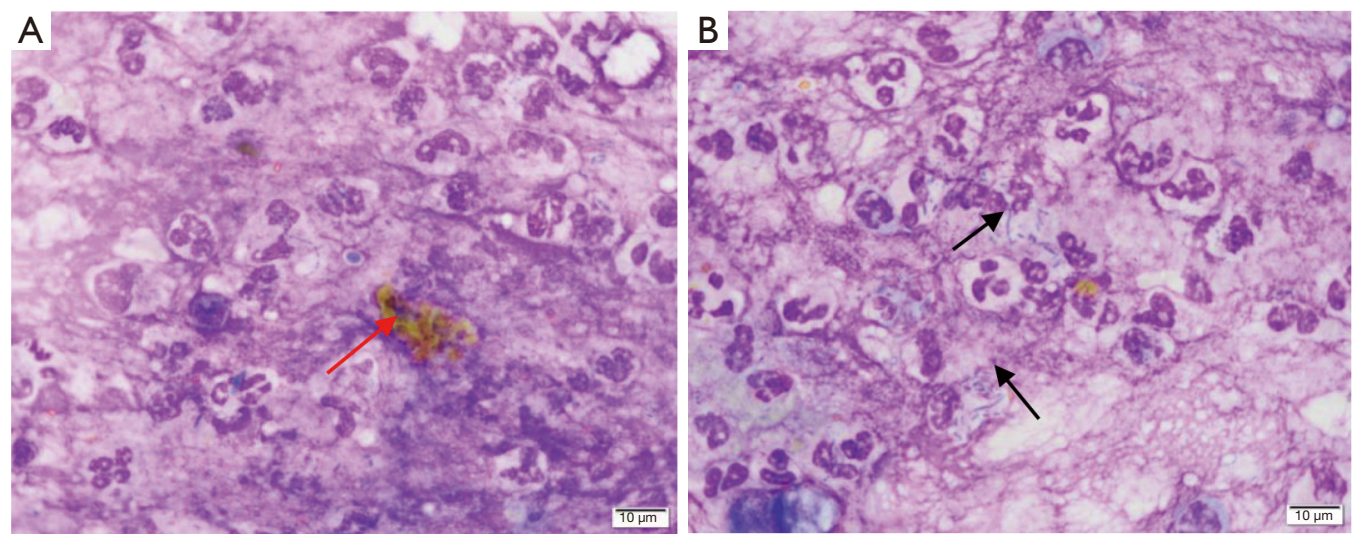

Figure 2 St. Regis staining of BALF smears ( $\times 1,000$ magnification). Bilirubin crystallization is shown by the red arrows and bacteria is shown by the black arrows. BALF, bronchoalveolar lavage fluid.

hepatic duct adenocarcinoma, moderately differentiated. In August 2017, she was admitted to our outpatient clinic, with "bile-like sputum after hepatocarcinoma surgery for more than 1 year". After admission, physical examination revealed a body temperature of $38.4{ }^{\circ} \mathrm{C}$, coarse breath sounds in both lungs, and obvious weight loss. Antiinfective treatment was administered and the relevant checks were improved. Routine BALF examination revealed that the BALF was yellow and turbid, the total number of cells was $5,000 \times 10^{6}$ cells/L, and the number of nucleated cells was $1,330 \times 10^{6}$ cells/L. The nucleated cells were classified as $92 \%$ neutrophils, $1 \%$ lymphocytes, and $7 \%$ macrophages. The BALF cytomorphology showed bilirubin crystallization and a large number of bacteria (Figure $2 A, B$ ). During hospitalization, both endoscopic retrograde cholangiopancreatography (ERCP) and bronchoscopy confirmed the right middle lobe BBF with infection. An electronic bronchoscope, chartis balloon exploration, freezedried human fibrinogen combined with silicone plugs, and EBV closure were performed. After the operation, the patient recovered well, and no biliptysis was observed. Postoperative anti-infective treatment was increase, and the patient's body temperature returned to normal. She was discharged 12 days after hospitalization with normal body temperature and no sputum was observed. A series of 3 follow-ups reported good recovery in the patient.

\section{Discussion}

$\mathrm{BBF}$ is a rare complication of hepatobiliary diseases. Since bile reflux can cause a severe chronic irritable cough, bronchopneumonia, dyspnea, and other severe 
complications such as necrotizing pneumonia, it should be diagnosed and treated as early as possible. Primary BBF is rare, and BBF is commonly secondary to diagnostic or treatment procedures such as hepatobiliary surgery, radiofrequency ablation of liver tumors, ERCPs, and percutaneous transhepatic cholangiographies (PTCs) in clinical practice $(4,5)$. At present, a specific diagnostic indicator for BBF is lacking. Clinically, the diagnosis of BBF relies on invasive operations such as cholangiography or surgical exploration, and improper selection of the puncture site or misoperation may cause iatrogenic BBF (6). Here, we reported bilirubin crystallization in the BALF of two BBF patients, suggesting that bilirubin crystallization can be used as an effective marker for the diagnosis of BBF. In addition, compared with the above-mentioned invasive procedures, BALF examination is simple and easy to perform, is less invasive and has good specificity. Therefore, it can be used as a routine examination for the differential diagnosis of $\mathrm{BBF}$ in clinical practice. However, this report only included two patients whose features could not represent those of the general population. Future studies with larger sample size are warranted to validate our findings.

In 1850, Peacock et al. (7) reported that a liver abscess in a patient with echinococcosis had abnormal traffic with the lung, and this was the first reported case of BBF in humans. In 2011, Liao et al. (4) reviewed and summarized 68 cases of patients with secondary BBF from the literatures published in the past 30 years. The results showed that $32.3 \%$ of $\mathrm{BBF}$ was secondary to tumors, and all patients had biliptysis, and the other symptoms included fever, cough, and jaundice. Almost half of the patients underwent endoscopic biliary stent drainage, and $41 \%$ of the patients received surgical treatment. Of the 28 patients who were followed up, $87.7 \%$ recovered at the end of follow-up. Among the 10 case reports of BFF (1-3,5,6,8-13) published over the past decade, only one was primary BBF (13). The other 9 cases were iatrogenic BBF, including 3 cases secondary to radiofrequency ablation for liver metastatic cancer $(2,3,9)$, 2 cases secondary to cholelithiasis $(1,8), 2$ cases secondary to partial hepatectomy $(10,12), 1$ case secondary to combined thoracic-abdominal surgery (5), 1 case secondary to pancreaticoduodenectomy (6), and 1 case secondary to trauma (11).

In previous case reports, coughing up bile-like sputum is a typical manifestation of most BBF patients. That is, bile is coughed up from the sputum through the pathological pathway, coughing "bile-like" sputum. Therefore, once a patient is found to cough up yellow or yellow-green bile- like sputum, and the patient has risk factors for BBF (such as biliary obstruction, hepatobiliary surgery, etc.) (9), a possible diagnosis of BBF must be considered. However, sometimes bile-like sputum is not easily distinguished from yellow sputum caused by infection (1), while the compositional examination of sputum is more specific. In the past, clinicians commonly relied on imaging and clinical manifestations to diagnose BBF, and there have been few reports on BALF examinations for BBF diagnosis. In this paper, the two patients both presented with cough and expectoration (bile-like sputum), and yellow lumps of bilirubin crystallization were detected in the cytomorphological examination of the BALF, which confirmed the bile components of the sputum and therefore contributed to the definite diagnosis of $\mathrm{BBF}$. The diagnosis was subsequently confirmed by MRCP and ERCP. Therefore, these findings suggested that bilirubin crystallization in BALF can be used as an important marker for the diagnosis or differential diagnosis of BBF. The routine cytomorphological examination of the sputum or BALF is simple, efficient, less-invasive, and accurate. Therefore, in clinical practice, routine cytomorphological examination of the sputum and BALF should be performed for patients suspected of BBF to improve diagnostic efficacy.

\section{Acknowledgments}

Funding: None.

\section{Footnote}

Reporting Checklist: The authors have completed the CARE reporting checklist. Available at https://dx.doi. org/10.21037/apm-21-1040

Conflicts of Interest: All authors have completed the ICMJE uniform disclosure form (available at https://dx.doi. org/10.21037/apm-21-1040). The authors have no conflicts of interest to declare.

Ethical Statement: The authors are accountable for all aspects of the work in ensuring that questions related to the accuracy or integrity of any part of the work are appropriately investigated and resolved. All procedures performed in studies involving human participants were in accordance with the ethical standards of the institutional and/or national research committee(s) and with the Helsinki Declaration (as revised in 2013). Written informed consent 
was obtained from the patient.

Open Access Statement: This is an Open Access article distributed in accordance with the Creative Commons Attribution-NonCommercial-NoDerivs 4.0 International License (CC BY-NC-ND 4.0), which permits the noncommercial replication and distribution of the article with the strict proviso that no changes or edits are made and the original work is properly cited (including links to both the formal publication through the relevant DOI and the license). See: https://creativecommons.org/licenses/by-nc-nd/4.0/.

\section{References}

1. Cheng AC, Chen HW, Chen PJ, et al. Bronchobiliary Fistula. Intern Emerg Med 2021;16:1093-4.

2. Huang $\mathrm{ZM}, \mathrm{Zuo} M \mathrm{MX}, \mathrm{Gu} \mathrm{YK}$, et al. Bronchobiliary fistula after ablation of hepatocellular carcinoma adjacent to the diaphragm: Case report and literature review. Thorac Cancer 2020;11:1233-8.

3. Xi XJ, Zhang Y, Yin YH, et al. Bronchobiliary fistula following radiofrequency ablation for liver metastases from breast cancer: A case report and literature review. Medicine (Baltimore) 2018;97:e12760.

4. Liao GQ, Wang H, Zhu GY, et al. Management of acquired bronchobiliary fistula: A systematic literature review of 68 cases published in 30 years. World J Gastroenterol 2011;17:3842-9.

5. Mauduit M, Rouze S, Turner K, et al. Combined thoracic and hepatobiliary surgery for iatrogenic bronchobiliary fistula. Asian Cardiovasc Thorac Ann 2018;26:63-6.

6. Odufalu FD, Zubairu J, Silverman W. Bronchobiliary fistula: a rare complication after pancreaticoduodenectomy. BMJ Case Rep 2018;2018:bcr2017221895.

7. Peacock TB. Case in Which Hydatids Were Expectorated, and One of Suppuration in a Hydatid Cyst of the Liver Communicating with the Lungs. Edinb Med Surg J 1850;74:33-46.

8. Martínez-Velado E, Palomar-Rodríguez LM, OlivoEsteban JR. Bronchobiliary fistula. Rev Esp Enferm Dig 2012;104:210-1.

9. Crnjac A, Pivec V, Ivanecz A. Thoracobiliary fistulas: literature review and a case report of fistula closure with omentum majus. Radiol Oncol 2013;47:77-85.

10. Hai S, Iimuro Y, Hirano T, et al. Bronchobiliary fistula caused after hepatectomy for hepatocellular carcinoma: a case report. Surg Case Rep 2016;2:147.

11. Banerjee N, Rattan A, Priyadarshini P, et al. Post-traumatic bronchobiliary fistula. BMJ Case Rep 2019;12:e228294.

12. Lazarou V, Moris D, Papalampros A, et al. Bronchobiliary fistula after hepatectomy: A case report and review of the literature. Mol Clin Oncol 2019;11:602-6.

13. Li TY, Zhang ZB. Congenital bronchobiliary fistula: A case report and review of the literature. World J Clin Cases 2019;7:881-90.

(English Language Editor: J. Teoh)
Cite this article as: Zhang N, Chen Y, Tang L, Zhou D, Hou T. Diagnosis of bronchobiliary fistula by bilirubin crystallization in the alveolar lavage fluid: case reports and literature review. Ann Palliat Med 2021;10(6):7121-7125. doi: 10.21037/apm-21-1040 